PROCEEDINGS OF THE

AMERICAN MATHEMATICAL SOCIETY

Volume 127, Number 2, February 1999, Pages 589-597

S 0002-9939(99)04995-3

\title{
CUT SETS AND NORMED COHOMOLOGY WITH APPLICATIONS TO PERCOLATION
}

\author{
ERIC BABSON AND ITAI BENJAMINI
}

(Communicated by Jeffry N. Kahn)

\begin{abstract}
We discuss an inequality for graphs, which relates the distances between components of any minimal cut set to the lengths of generators for the homology of the graph. Our motivation arises from percolation theory. In particular this result is applied to Cayley graphs of finite presentations of groups with one end, where it gives an exponential bound on the number of minimal cut sets, and thereby shows that the critical probability for percolation on these graphs is neither zero nor one. We further show for this same class of graphs that the critical probability for the coalescence of all infinite components into a single one is neither zero nor one.
\end{abstract}

\section{INTRODUCTION}

Let $G=(V, E)$ be a locally finite graph. Given two vertices $u, v \in V$, a $(u, v)$ cut set $\Pi \subseteq E$ is a set of edges that has nonempty intersection with every path from $u$ to $v$. Similarly a $(u, \infty)$ cut set intersects every path from $u$ contained in no finite subgraph. A $(u, v)$ cut set is minimal (an mcs) if it contains no proper subset which is also a $(u, v)$ cut set.

Cut sets are naturally connected to homology. We will show how the distance between the two parts of any bipartition of a minimal cut set is related to the $L_{\infty}$ norm on the lattice of integral first cohomology classes and hence also to the $L_{1}$ norm on homology. In particular we show that no bipartition of a minimal cut set can have its two parts separated by a distance of more than half the diameter of the first homology lattice. For Cayley graphs, this diameter is in turn related to the lengths of the relators. We present the inequality in the context of locally compact, complete, path metric spaces, associating to a graph the path metric in which the length of every edge is one.

Our motivation comes from percolation theory [1], [3]. To date percolation has mainly been studied on $\mathbb{Z}^{d}$, the Cayley graph for the free Abelian group with $d$ free generators. The basic notion in percolation theory is $p_{c}(G)$, the critical probability for bond percolation on the graph $G$. In Bernoulli bond percolation the edges are independently colored open with probability $p$. Those edges that are not open are colored closed. The corresponding product measure on the edge colorings is denoted $P_{p, G}$ or $P_{p}$. For a fixed open/closed edge coloring $E_{o} \cup E_{c}=E$ of a graph $G$ with

Received by the editors March 13, 1997.

1991 Mathematics Subject Classification. Primary 60K35.

(C)1999 American Mathematical Society 
a vertex $v$ define the cluster of $v$ to be

$$
\left.C(v) \stackrel{\text { def }}{=} \text { (the connected component containing } v \text { of the subgraph }\left(V, E_{o}\right) \text { of } G\right) \text {. }
$$

Write

$$
\theta_{v}(p) \stackrel{\text { def }}{=} P_{p}[C(v) \text { is infinite }]
$$

for the probability that $C(v)$ is infinite if edges of $G$ are independently colored open with probability $p$. On a Cayley graph (or any graph with a vertex transitive group action), the value of $\theta_{v}(p)$ is independent of the choice of $v$, and hence the subscript $v$ is dropped. If $C(v)$ is infinite, we say that percolation occurs from $v$. Let

$$
p_{c} \stackrel{\text { def }}{=} \inf \{p \mid \theta(p)>0\}
$$

be the critical probability for percolation. This will be independent of the vertex $v$ if the graph $G$ is connected. Site percolation is defined similarly, with vertex coloring replacing edge coloring. These are closely related, but we will use only bond percolation. See [3] for more information about percolation. Another critical probability which we will use in the final section is the critical probability for the infinite cluster to be unique. Let

$$
\left.p_{u} \stackrel{\text { def }}{=} \inf \left\{p \mid P_{p} \text { [there is exactly one infinite cluster }\right]=1\right\} .
$$

The fundamental theorem of percolation theory states that if $d \geq 2$, then $0<$ $p_{c}\left(\mathbb{Z}^{d}\right)<1$. To initiate the study of percolation on other graphs (see [1]), we would like to start with this result. First, it is standard $[3,1.4]$ that $p_{c}(G) \geq d^{-1}$ for any infinite graph $G$ with every vertex contained in at most $d$ edges (e.g. Cayley graphs for infinite groups with up to $d$ generators). This paper will concentrate on finding conditions under which equation (0) below holds for some vertex $v$ and constant $r$, since by a Peierls argument $[3,1.4]$ this implies that $p_{c}(G)<1-r^{-1}$.

(0)

For every $n$ (the number of $(v, \infty)$ minimal cut sets in $G$ with $n$ elements) $\leq r^{n}$.

Note that this fails for $\mathbb{Z}$, where there are infinitely many size 2 minimal cut sets, and none of any other size. In this case $p_{c}(\mathbb{Z})=1$.

Conjecture 0. If $\Gamma$ is a finitely generated group which is not a finite extension of $\mathbb{Z}$ or $\mathbb{Z}_{2} \star \mathbb{Z}_{2}$ (the infinite dihedral group), then equation (0) holds for the Cayley graph of $\Gamma$.

As a corollary to the main inequality in this paper we get

Theorem 9. If $\Gamma$ is a finitely presented group which is not a finite extension of $\mathbb{Z}$ or $\mathbb{Z}_{2} \star \mathbb{Z}_{2}$ (the infinite dihedral group), then equation (0) holds for the Cayley graph of $\Gamma$.

Note that the case where the number of ends is not one is known (see the proof of Theorem 9).

This paper is organized as follows. Section 1 contains definitions and notation in the context of path metric spaces. Section 2 presents the main inequality. Section 3 describes a family of examples showing that the inequality from section 2 can be strict. Section 4 applies the inequality to graphs, proving Conjecture 0 for finitely presented groups. Section 5 gives a non-triviality condition for $p_{u}$ similar to that of section 4 for $p_{c}$. 
We would like to thank Clara Chan, Oded Schramm and our referee for thoroughly reading previous drafts and making many useful suggestions.

\section{Definitions And NOtation}

A metric space $X$ is called a path metric space if there is a continuous additive function $l$ from paths in $X$ to $\mathbb{R}_{\geq 0}$, called the length of the path, such that the distance between any two points is equal to the infimum of the lengths of paths joining these points.

In what follows we will assume that $X$ is a locally compact, complete, path metric space.

Define $X_{+}$to be the compactification of $X$ in which two sequences in $X$ converge to the same point in $X_{+}$iff for every proper map from $X$ to $(0,1)$ the images converge to the same point in $[0,1]$. (Note that a proper map is a continuous map in which all inverse images of compact sets are compact.) The points of $X_{+} \backslash X$ are called the ends of $X$ or the connected components of $X$ at infinity [8]. Extend the metric by setting $d(z, x)=\infty$ if $z \in X_{+} \backslash X$ and $x \neq z$. Call $X$ connected at infinity if $X$ has one end or equivalently if $X_{+}$is the one point compactification of $X$. For instance $\mathbb{R}$ has two ends, so $\mathbb{R}_{+}$is homeomorphic to a closed line segment, while $\mathbb{R}^{2}$ has one end, so $\left(\mathbb{R}^{2}\right)_{+}$is a sphere.

Denote by $H_{1} X$ the first homology group and by $H^{1} X$ the first cohomology group of $X$ with coefficients in $\mathbb{Z}$. For any map $\psi: X \rightarrow S^{1}$, let

$$
[\psi] \stackrel{\text { def }}{=} \psi^{*} 1 \in H^{1} X
$$

where 1 is a fixed generator of $H^{1} S^{1}=\mathbb{Z}$. Similarly, for any map $\varphi: S^{1} \rightarrow X$, let

$$
[\varphi] \stackrel{\text { def }}{=} \varphi_{*} \hat{1} \in H_{1} X
$$

where $\hat{1}$ is the generator of $H_{1} S^{1}=\mathbb{Z}$ dual to the 1 above. See [7] for topological definitions, and note that $\operatorname{Hom}\left(H_{1} X, \mathbb{Z}\right)=H^{1} X$ is free.

We next define the infinity norm on $H^{1} X$ and the dual one norm on $H_{1} X$, and use these to give two measures of the sparseness of the lattice $H_{1} X ; L_{X}$ will be the maximum distance between two parallel, adjacent, codimension one, affine sublattices in $H_{1} X$, while $K_{X}$ will be the minimum radius of a ball about 0 containing a rational basis for $H_{1} X$.

Let $S^{1}=\left[-\frac{1}{2}, \frac{1}{2}\right] /\left(-\frac{1}{2} \sim \frac{1}{2}\right)$ inherit from the interval the path metric in which antipodal points are at a distance of $\frac{1}{2}$. Given a map $\psi: X \rightarrow S^{1}$, denote by $\|\psi\|_{\infty}$, the Lipschitz constant of the map, that is:

$$
\|\psi\|_{\infty} \stackrel{\text { def }}{=} \sup _{x \neq y \in X} \frac{d(\psi(x), \psi(y))}{d(x, y)} .
$$

For $\sigma \in H^{1} X$ let

$$
\|\sigma\|_{\infty} \stackrel{\text { def }}{=} \inf \left\{\|\psi\|_{\infty} \mid[\psi]=\sigma\right\}
$$

Let

$$
L_{X} \stackrel{\text { def }}{=} \sup \left\{\|\sigma\|_{\infty}^{-1} \mid \sigma \in H^{1} X \backslash\{0\}\right\} .
$$

Note that $\|\sigma\|_{\infty}=0$ iff $\sigma=0$. If $H^{1} X=\{0\}$, then let $L_{X}=0$. 
If $\varphi: S^{1} \rightarrow X$, then let $\|\varphi\|_{1} \stackrel{\text { def }}{=} l\left(\varphi^{\prime}\right)$ where $\varphi^{\prime}:[0,1] \rightarrow X$ is the path in $X$ given by $\varphi^{\prime}(x)=\varphi\left(x-\frac{1}{2}\right)$ and $l$ is the length function in $X$. If $h \in H_{1} X$, let

$$
\begin{aligned}
\|h\|_{1} \stackrel{\text { def }}{=} \inf \left\{\sum_{i} a_{i}\left\|\varphi_{i}\right\|_{1} \mid \varphi_{i}: S^{1} \rightarrow X\right. \text { and } \\
\left.\qquad a_{i} \in \mathbb{Q} \text { such that } \sum_{i}\left[\varphi_{i}\right] \otimes a_{i}=h \otimes 1\right\} .
\end{aligned}
$$

Let

$$
\begin{aligned}
& K_{X} \stackrel{\text { def }}{=} \inf \left\{t \mid \text { there are } h_{i} \in H_{1} X\right. \text { with } \\
& \left.\qquad H_{1} X \otimes \mathbb{Q}=\operatorname{span}\left\{h_{i} \otimes 1\right\} \text { and }\left\|h_{i}\right\|_{1} \leq t\right\} .
\end{aligned}
$$

If $H_{1} X \otimes \mathbb{Q}=\{0\}$, then set $K_{X}=0$.

We turn now to the notion of minimal cut set in a path metric space. If $X$ is a locally compact, complete, path metric space, with $x \neq y \in X_{+}$, then an $(x, y)$ minimal cut set (mcs) $\Pi$ is a compact subset of $X \backslash\{x, y\}$, satisfying the following two conditions.

(1) (cut set) Every path $\gamma$ from $x$ to $y\left(\gamma:[0,1] \rightarrow X_{+}\right.$with $\gamma(0)=x$ and $\gamma(1)=y)$ has $\gamma([0,1]) \cap \Pi \neq \varnothing$.

(2) (minimality) $\Pi$ is equal to the intersection of the closures of the two path components of $X_{+} \backslash \Pi$ containing $x$ and $y$ respectively.

Note that a closed subset $\Pi$ of $X_{+}$satisfies (1) iff there is a continuous map $\tau: X_{+} \rightarrow \mathbb{R}_{+}$with $\tau(x)<0, \tau(y)>0$, and $\Pi=\tau^{-1}(0)$. To see this, set $\tau(z)=$ $d(z, \Pi)$ if $z$ is in the path component of $X_{+} \backslash \Pi$ containing $y$, and $\tau(z)=-d(z, \Pi)$ otherwise.

Note also that every cut set contains some minimal cut set. In particular, if $\Pi \subseteq X$ satisfies (1), then $\Pi^{\prime \prime} \subseteq \Pi$ satisfying (1) and (2) can be directly constructed in two steps: Let $\Pi^{\prime}=\bar{Y} \backslash Y$, where $Y$ is the path component of $X_{+} \backslash \Pi$ containing $y$. Then let $\Pi^{\prime \prime}=\bar{Z} \backslash Z$, where $Z$ is the path component of $X_{+} \backslash \Pi^{\prime}$ containing $x$.

We would like to analyze the distance between components in a bipartition of a minimal cut set. Given a nonempty set $Y \subseteq X$, let

$$
C(Y)=\sup \left\{d\left(Y_{1}, Y_{2}\right) \mid Y_{1} \cup Y_{2}=Y\right\} .
$$

If $C(Y) \leq t$, then $Y$ is said to be $t$-close. Let

$$
C_{X}=\sup \left\{C(\Pi) \mid \Pi \text { an }(x, y) \text {-mcs for some points } x \neq y \in X_{+}\right\} \text {. }
$$

\section{MAin RESUlt}

Theorem 1. If $X$ is a locally compact, complete, path metric space, then $2 C_{X} \leq$ $K_{X}$.

Proof. The theorem is a direct consequence of the following two lemmata.

Lemma 2. If $X$ is a locally compact, complete, path metric space, then $L_{X} \leq K_{X}$.

Proof. If $H^{1} X=\{0\}$, then $L_{X}=0$; otherwise choose $\sigma \in H^{1} X \backslash\{0\}$. Since $H^{1} X=\operatorname{Hom}\left(H_{1} X, \mathbb{Z}\right)$ is free and the norms $\|\cdot\|_{1}$ on $H_{1} X \otimes \mathbb{Q}$ and $\|\cdot\|_{\infty}$ on $H^{1} X \otimes \mathbb{Q}$ are dual [5, p. 16], $\|\sigma\|_{\infty}\|h\|_{1} \geq|h \cdot \sigma|$ for all $h \in H_{1} X$. If $H_{1} X \otimes \mathbb{Q}=\operatorname{span}\left\{h_{i} \otimes 1\right\}$ with $h_{i} \in H_{1} X$, then $h_{i} \cdot \sigma \neq 0$ for some $i$ and hence $\|\sigma\|_{\infty}^{-1} \leq \frac{\left\|h_{i}\right\|_{1}}{\left|h_{i} \cdot \sigma\right|} \leq K_{X}$.

Lemma 3. If $X$ is a locally compact, complete, path metric space, then $2 C_{X} \leq L_{X}$. 
Proof. We will assume that $C_{X}$ is nonzero, choose a minimal cut set witnessing this, and construct from it a nonzero cohomology class with small infinity norm. If $C_{X}=0$ (which we will show must be the case if $H^{1} X=\{0\}$ ) the inequality holds trivially.

Assume that $C_{X}>0$. Choose $\Pi=\Pi_{1} \cup \Pi_{2}$ an $(x, y)$-mcs with $d\left(\Pi_{1}, \Pi_{2}\right)>0$ and define $\varphi: X \rightarrow S^{1}=\left[-\frac{1}{2}, \frac{1}{2}\right] /\left(-\frac{1}{2} \sim \frac{1}{2}\right)$ by first writing

$$
X=\Pi \cup X_{x} \cup X_{y}
$$

where $X_{x}$ is the path connected component of $X \backslash \Pi$ having $x \in \bar{X}_{x} \subseteq X_{+}$, and $X_{y}$ is the union of the remaining path components of $X \backslash \Pi$. Then let

$$
\varphi(z)=\frac{\varepsilon}{2} \min \left\{\frac{d\left(z, \Pi_{1}\right)}{d\left(\Pi_{1}, \Pi_{2}\right)}, 1\right\},
$$

where $\varepsilon=1$ if $z \in \Pi \cup X_{x}$ and $\varepsilon=-1$ if $z \in X_{y}$.

To see that $[\varphi] \in H^{1} X$ is nontrivial, first choose points $x^{\prime}$ and $y^{\prime}$ in $X$ which are in the same connected component in $X_{+} \backslash \Pi$ as $x$ and $y$ respectively, then choose paths $\gamma_{i}$ from $x^{\prime}$ to $y^{\prime}$ in $X \backslash \Pi_{i}$. The paths $\gamma_{i}$ exist by the minimality of $\Pi$. More explicitly, if $\pi \in \Pi_{1}$, then since $X$ is a path space and $d\left(\Pi_{1}, \Pi_{2}\right)>0$ there is a small path connected ball $B \subset X \backslash \Pi_{2}$ containing $\pi$. Since $\pi$ is in the closure of $X_{x}$, there is $x^{\prime \prime} \in B \cap X_{x}$; hence there is a path in $X \backslash \Pi_{2}$ from $x^{\prime}$ to $\pi$. Similarly there is a path in $X \backslash \Pi_{2}$ from $y^{\prime}$ to $\pi$. These two combine to give $\gamma_{2}$. Combining the paths $\gamma_{1}$ and $\gamma_{2}$ gives a map $\gamma: S^{1} \rightarrow X$ with $\varphi \circ \gamma: S^{1} \rightarrow S^{1}$ having degree one. Thus $[\varphi] \neq 0$; hence $H^{1} X \neq\{0\}$.

It only remains to bound $\|\varphi\|_{\infty}$. This will be done by two cases.

I) If $\{z, w\} \subseteq \Pi \cup X_{x}$ or $\{z, w\} \subseteq \Pi \cup X_{y}$, then

$$
2 d\left(\Pi_{1}, \Pi_{2}\right) d(\varphi(z), \varphi(w)) \leq\left|d\left(z, \Pi_{1}\right)-d\left(w, \Pi_{1}\right)\right| \leq d(z, w)
$$

where the first inequality follows from the definition of $\varphi$ and the second from the triangle inequality.

II) If $z \in X_{x}$ and $w \in X_{y}$, then since every path $\gamma$ from $z$ to $w$ intersects $\Pi$,

$$
\begin{aligned}
& 2 d\left(\Pi_{1}, \Pi_{2}\right) d(\varphi(z), \varphi(w)) \\
& \quad \leq \min \left(d\left(z, \Pi_{1}\right)+d\left(w, \Pi_{1}\right), d\left(z, \Pi_{2}\right)+d\left(w, \Pi_{2}\right)\right) \leq l(\gamma) .
\end{aligned}
$$

Hence since $X$ is a path space $2 d\left(\Pi_{1}, \Pi_{2}\right) d(\varphi(z), \varphi(w)) \leq d(z, w)$ in both cases. Thus $2 d\left(\Pi_{1}, \Pi_{2}\right) \leq\|\varphi\|_{\infty}^{-1}$, and the lemma follows.

\section{An example}

Sometimes $L_{X}<K_{X}$. One source of examples is to choose a full rank lattice $T$ in the Lie group $\mathbb{R}^{n}$, and take $X=\mathbb{R}^{n} / T$ to be the compact $n$-torus with the quotient metric. Since $T$ is Abelian and acts freely, $H_{1} X=T$ and it turns out that the norm $\|\cdot\|_{1}$ is the $L_{2}$ norm on $\mathbb{R}^{n}$ restricted to $T$. Now $K_{X}$ is the minimum size of a Euclidean ball containing enough elements of $T$ to span $\mathbb{R}^{n}$, while $L_{X}$ is the maximum distance between parallel codimension one affine subspaces spanned by elements of $T$. If $T$ has an orthogonal basis these are the same. Now choose $n=2$ and $T=\langle(2,0),(1,2)\rangle$, and check that $L_{X}=2$ and $K_{X}=\sqrt{5}$. 


\section{Minimal CUt Sets in GRAPhS}

Given a locally finite graph $G=(V, E)$, let $G$ also denote the path metric space obtained by identifying the edges of $G$ with intervals of length one. Write $E$ also for the set of edge midpoints of $G$. Note that a graph minimal cut set as defined in the introduction is equivalent to a path space minimal cut set $\Pi$ for $G$ with $\Pi \subseteq E$.

Corollary 4. If $\Pi$ is a graph minimal cut set in $G$, then $2 C(\Pi) \leq K_{G}$.

We now define some auxiliary graphs: Define the graph $G^{t}$ to have vertices $V$, and an edge $\{x, y\}$ if $d_{G}(x, y) \leq t$. Define the graph $G_{E}$ to have vertices $E$, and an edge $\{e, f\}$ if $e$ and $f$ share a vertex in $G$. Thus any $t$-close set of edge midpoints in $G$ is a $t$-close set of vertices in $G_{E}$ and a connected set of vertices in $\left(G_{E}\right)^{t}$. Let $B_{G}(x, r) \stackrel{\text { def }}{=}\{v \in V \mid d(v, x) \leq r\}$. Let

$$
\begin{aligned}
R(G, t) & \stackrel{\text { def }}{=} \max _{v \in V}\left(\text { the valence of } v \text { in } G^{t}\right) \\
& =\max _{v \in V}\left(\text { the number of elements in } B_{G}(v, t)\right) .
\end{aligned}
$$

Let $N_{u, v}^{n}(G)$ be the number of $(u, v)$ graph minimal cut sets in $G$ containing exactly $n$ edge midpoints.

The closeness of a graph mcs is approximately the same as for an arbitrary mcs. More precisely:

Note 5. If $x$ and $y$ are vertices of $G$ or infinite points in $G_{+}$, then

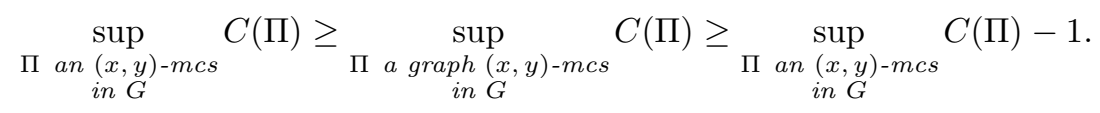

Proof. Fix an $(x, y)$-mes $\Pi$ in $G$ and define $\Pi^{\prime}=B_{G_{E}}\left(\Pi, \frac{1}{2}\right)$. Now $\Pi^{\prime}$ is an $(x, y)$ cut set in $G$, which contains an $(x, y)$-mcs $\Pi^{\prime \prime} \subseteq \Pi^{\prime}$, with $C(\Pi)+1 \geq C\left(\Pi^{\prime \prime}\right) \geq$ $C(\Pi)-1$.

Lemma 6. If $G$ is a graph with a vertex $v$, then there are at most $R(G, t)^{2 n}$ subsets of $V$ which are $t$-close, have $n$ elements, and contain $v$.

Proof. Take $U$ to be a $t$-close $n$-element subset of $V$ containing $v$. Since $U$ is $t$-close, the restriction of $G^{t}$ to $U$ is connected, and hence we can choose a spanning tree $T$ for this graph, and thence a path in $G^{t}$ beginning at $v$ with length $2 n$ and support $T$. Since the set $U$ can be recovered from this path, it suffices to note that there are at most $R(G, t)^{2 n}$ paths in $G^{t}$ with length $2 n$ beginning at $v$.

Proposition 7. If $G$ is a graph with vertices and $u$ and $v$, then

$$
N_{u, v}^{n}(G) \leq d(v, u)\left(R\left(G_{E}, \frac{K_{G}}{2}\right)\right)^{2 n} .
$$

Proof. Let $\gamma$ be a path from $u$ to $v$ of length $d(u, v)$. Any graph $(u, v)$-mcs $\Pi$, intersects $\gamma$ and is $\frac{K_{G}}{2}$-close. Thus the bound follows from Lemma 6 .

Proposition 8. If $G$ is a graph with one end, $u$ is a vertex, $v$ is either a vertex or the infinite point in $G_{+}$and there is a bi-infinite geodesic through $u$ and $v$, then

$$
N_{u, v}^{n}(G) \leq n K_{G}\left(R\left(G_{E}, \frac{K_{G}}{2}\right)\right)^{2 n} .
$$


Proof. Assume that $v$ is a vertex. Since $G$ is connected at infinity, every $(u, v)$ mcs must intersect both the geodesic segment between $u$ and $v$ and one of the two unbounded geodesics either from $u$ or from $v$. Since every minimal cut set is $\frac{K_{G}}{2}$ close, every minimal cut set with at most $n$ elements has every pair of elements at most $n \frac{K_{G}}{2}$ apart. Thus there must either be two intersections between the cut set and the geodesic within $n \frac{K_{G}}{2}$ of $u$ or within $n \frac{K_{G}}{2}$ of $v$. Applying Lemma 6 gives the bound.

If $v$ is the infinite point, then both intersection points must be within $n \frac{K_{G}}{2}$ of $u$, and we actually get a better bound by a factor of two.

Example 1. If $G$ is a tree, then $K_{G}=0$.

Example 2. If $G$ is the Cayley graph for $\mathbb{Z}^{d}$ with $d \geq 2$ generators, then $K_{G}=4$ and $R\left(G_{E}, 2\right)=3-6 d+8 d^{2}$.

Example 3. If $G$ is the Cayley graph of any finitely generated group

$$
\left\langle g_{1}, \ldots, g_{m} \mid R\right\rangle,
$$

then

$$
K_{G} \leq \max _{r \in R}\{\text { length of the cyclic reduction of the relation } r\}
$$

and $R\left(G_{E}, \frac{K_{G}}{2}\right) \leq 2(2 m)^{\frac{K_{G}}{2}}$.

Theorem 9. If $\Gamma$ is a finitely presented group which is not a finite extension of $\mathbb{Z}$ or $\mathbb{Z}_{2} \star \mathbb{Z}_{2}$ (the infinite dihedral group), then equation (0) holds for the Cayley graph of $\Gamma$.

Proof. Take $\Gamma$ to be a finitely presented group with $k$ generators, and write $G=$ $(V, E)$ for the Cayley graph of $\Gamma$, with a fixed vertex $v \in V$. If $\Gamma$ is not connected at infinity, the theorem is known.

Groups with zero ends are finite, so percolation does not make sense and equation (0) holds trivially.

Groups with two ends are finite extensions of $\mathbb{Z}$ or $\mathbb{Z}_{2} \star \mathbb{Z}_{2}$ [8, 4.A.6.5], (so $p_{c}=1$ ).

Groups with more than two ends contain a copy of $\mathbb{Z} \star \mathbb{Z}[8,5$. A.10], and are thus not amenable $[6,2.2 .5]$ since $\mathbb{Z} \star \mathbb{Z}$ lacks an invariant mean. That non-amenable groups satisfy equation (0) can be seen by noting that if $G$ is the Cayley graph of a non-amenable group, then there is a constant $k_{G}$ such that for every $A \subseteq V$ with $|A|$ elements, the number of edges with exactly one end in $A$ is always at least $k_{G}|A|$. Thus for a $(v, \infty)$ minimal cut set $\Pi$ with $n$ edges, the number of vertices in the connected component of $G \backslash \Pi$ which contains $v$ is at most $k_{G}^{-1} n$, and hence the number of possible cut sets of size $n$ is at most the number of connected subgraphs of $G$ containing $v$ and having at most $k_{G}^{-1} n$ vertices, which by Lemma 6 for a group with $m$ generators is at most $(2 m)^{2 k_{G}^{-1} n}$.

Finally, if $\Gamma$ is connected at infinity and has a bi-infinite geodesic through $v$, then by Example 3 and Proposition 8 we get $N_{v, \infty}^{n}(G) \leq 2 n K_{G}\left(4(2 k)^{K_{G}}\right)^{n}$. Thus it only remains to show that every infinite Cayley graph has some bi-infinite geodesic through $v$. This can be done, using the action of the group $\Gamma$ on the graph $G$, by choosing any infinite sequence of distinct geodesics through $v$ and translating each of them so that $v$ is within $\frac{1}{2}$ of each center. Now since every finite diameter ball is finite, we can choose successive infinite subsequences which agree in arbitrarily large balls. 


\section{ON THE UNIQUENESS OF THE INFINITE PERCOLATION CLUSTER}

For percolation on $\mathbb{Z}^{d}$, if $\theta(p)>0$, then there exists, with probability one, a unique infinite open cluster (connected component of the subgraph with the edges colored open). See [3] and the elegant proof in [2]. As was shown in [4], for percolation on a ( $k$-regular tree) $\times \mathbb{Z}$, this is not the case if $k$ is sufficiently large. For some values of $p$, uniqueness holds, while for others, there are infinitely many infinite clusters. Benjamini and Schramm [1] defined

$$
\left.p_{u} \stackrel{\text { def }}{=} \inf \left\{p \mid P_{p} \text { [there is exactly one infinite cluster }\right]=1\right\},
$$

conjectured that $p_{c}<p_{u}$ for percolation on non-amenable Cayley graphs, and asked whether $p_{u}<1$ for transitive graphs with one end. Here we show the second inequality for Cayley graphs of finitely presented groups.

Theorem 10. For Cayley graphs of finitely presented groups with one end, $p_{u}<1$.

Proof. Fix a finitely presented group $\Gamma$ with one end, and a Cayley graph $G$. Fix $t$ such that every minimal cut set in $G$ is $t$-close, and choose $p$ with $1>p>$ $\max \left\{1-p_{c}\left(G^{t}\right), p_{c}(G)\right\}$. Now

$P_{p, G}$ [there are at least two infinite components of the open subgraph]

$\leq P_{p, G}$ [there is an infinite graph minimal cut set in $G$ with all edges closed]

$\leq P_{p, G}$ [there is an infinite $t$-close set of closed edges]

$\leq P_{p, G^{t}}\left[G^{t}\right.$ has an infinite closed cluster $]=0$.

The first inequality follows from the fact that if $u \in C_{1}$ and $v \in C_{2}$ are two infinite open clusters in a certain coloring of $G$, then there is a graph $(u, v)$-cut set, hence mcs, containing only closed edges. This cut set must be infinite since $G$ is connected at infinity, and $C_{1}$ and $C_{2}$ are infinite. The second inequality follows from the fact that minimal cut sets in $G$ are $t$-close. The final inequality follows from the observation that one way to sample from $P_{p, G^{t}}$ is to choose a coloring in $P_{p, G}$ and then add the remaining edges independently. The final equality follows from the fact $p>1-p_{c}\left(G^{t}\right)$.

Thus with $p$ chosen as above there is probability one of either one or zero infinite components, but since $p>p_{c}(G)$ there is probability one of having at least one infinite component.

\section{QUESTIONS}

Question 1. Does having some exponential bound on the number of minimal cut sets of size $n$ in a Cayley graph depend only on the group and not on the choice of generators?

Question 2. Does the property " $C_{G}$ is finite" for a Cayley graph depend only on the group and not on the choice of generators?

Question 3. Are there finitely generated groups with one end so that $C_{G}$ is not finite? Any such group must be infinitely presented. 


\section{REFERENCES}

1. I. Benjamini and O. Schramm, Percolation beyond $\mathbb{Z}^{d}$, many questions and a few answers, Electronic Communications in Probability 1 (1996), 71-82. MR 97j:60179

2. R. Burton and M. Keane, Density and uniqueness in percolation, Comm. Math. Phys. 121 (1989), 501-505. MR 90g:60090

3. G. Grimmett, Percolation, Springer-Verlag, New York, 1989. MR 90j:60109

4. G. Grimmett and C. Newman, Percolation in $\infty+1$ dimensions, in Disorder in physical systems, (G. R. Grimmett and D. J. A. Welsh, eds.), Clarendon Press, Oxford, 1990, pp. 219-240. MR 92a:60207

5. M. Gromov, Volume and bounded cohomology, Publication Mathématiques IHES 56 S-100 (1982). MR 84h:53053

6. A. Lubotzky, Discrete Groups, Expanding Graphs and Invariant Measures, Birkhäuser Verlag, Basel, 1994. MR 96g:22018

7. E. Spanier, Algebraic Topology, McGraw-Hill, Springer-Verlag, 1966, 1982. MR 35:1007; MR 83i:55001

8. J. Stallings, Group theory and three-dimensional manifolds, Yale University Press, New Haven 1971. MR 54:3705

Department of Mathematics, University of Washington, Box 354350, Seattle, WashINGTON 98195-4350

E-mail address: babson@math.ias.edu

Department of Mathematics, The Weizmann Institute, Rehovot 76100, Israel

E-mail address: itai@wisdom.weizmann.ac.il 